23

\title{
Исследование метода локальной оптической томографии по дифференциальным проекциям
}

\author{
(C) Г.Н. Вишняков, Г.Г. Левин, В.Л. Минаев, М.М. Ермаков \\ Всероссийский научно-исследовательский институт оптико-фризических измерений, \\ 119361 Москва, Россия \\ e-mail: vish@vniiofi.ru
}

Поступила в редакцию 04.06.2018 г.

\begin{abstract}
Предложено использовать локальную томографию по дифференциальным (DIC) проекциям для оптических исследований внутренней структуры прозрачных объектов. Преимущество локальной томографии состоит в том, что она дает возможность быстрого вычисления искомого распределения в интересующей точке или области без проведения полной реконструкции всего сечения. Для получения количественных DIC-проекций разработан томографический микроскоп с интерферометром поперечного сдвига, в котором реализован метод фазовых шагов для восстановления фазы. Предложена процедура нормализации проекционных данных по геометрическим моментам от DIC-проекций. Теоретически показано и подтверждено компьютерным моделированием, что суммарное изображение из DIC-проекций пропорционально преобразованию Гильберта от исходной функции, описывающей объект, а применение к этому изображению обратного преобразования Гильберта ведет к восстановлению томограммы объекта. Приведены результаты реконструкции томограммы шарика из диоксида кремния по его экспериментальным DIC-проекциям. Сравнение результатов моделирования с результатами эксперимента показало их хорошее сходство.
\end{abstract}

DOI: $10.21883 /$ OS.2018.12.46952.155-18

\section{Введение}

Оптически прозрачные объекты широко распространены и востребованы в различных областях науки и техники. К таким объектам можно отнести стекловолокна, граданы, оптические микрорезонаторы, биологические клетки. В последнее время также стали появляться оптически прозрачные детали, изготовленные из стекла или полимера с использованием методов аддитивных технологий [1]. Например, на 3D-принтере в работе [2] из прозрачного полимера изготовлены сферическая линза и сканирующее зеркало для системы оптической когерентной томографии, а в работе [3] из плавленого кварца изготовлена миниатюрная цилиндрическая линза. Для всех прозрачных объектов требуется измерять не только форму и структуру поверхности, но также и неоднородность материала внутри изделия, которая может быть вызвана как внутренними напряжениями в случае изделий аддитивных технологий, так и различной плотностью составных частей объекта у живых клеток. Эти неоднородности описываются 3D-пространственным распределением показателя преломления. Для его измерения используются методы вычислительной томографии в сочетании с различными методами визуализации и регистрации проекционных данных, например, интерференционной микроскопией [4-9], интегральной фотоупругостью для реконструкции остаточных напряжений [10]. Световая волна, прошедшая через прозрачный объект, модулируется по фазе, и она несет информацию об оптической разности хода (ОРХ) излучения, прошедшего через объект. Для получения проекционных данных объект чаще всего зондируется параллельным пучком света по различным угловым направлениям.
Ранее в нашей работе [11] была предложена идея локальной томографии фазовых объектов по дифференциальным проекциям. Этот метод локальной томографии позволяет восстанавливать томограммы только тех областей объекта, которые представляют интерес. В сочетании с методом дифференциального интерференционного контраста (differential interference contrast, далее по тексту DIC), используемого для количественной визуализации проекционных данных, этот метод позволяет исследовать динамику отдельных внутренних органелл живой клетки за счет сокращения времени съема данных.

В настоящей работе на основании математического моделирования и экспериментальных работ проведено исследование метода локальной томографии. Экспериментальные работы выполнены на макете томографического фазового микроскопа, а в качестве тест-объекта использованы калиброванные шарики из диоксида кремния.

\section{Томограмма из DIC-проекций}

Пусть исследуемый объект описывается функцией $f(x, y, z)=n(x, y, z)-n_{0}, \quad$ где $n(x, y, z)$ - 3D-пространственное распределение показателя преломления объекта в декартовых координатах $(x, y, z), n_{0}$ - показатель преломления окружающей (иммерсионной) среды. В настоящей работе используется схема зондирования объекта, в которой волновые векторы зондирующих плоских пучков лежат в одной плоскости $(x, z)$, где ось $z$ совпадает с оптической осью микроскопа. При такой схеме зондирования исследуемый объект можно представить в виде отдельных параллельных слоев $y=$ const, 
и задача реконструкции $3 \mathrm{D}$-изображения $f(x, y, z)$ сводится к набору задач реконструкции 2D-томограмм $f(x, y=$ const, $z)$. Поэтому далее будем рассматривать задачу реконструкции 2D-изображения $f(x, z)$.

В классической томографии для реконструкции 2Dфункции $f(x, z)$ используются 1D-проекции:

$$
p(r ; \varphi)=\iint_{-\infty}^{\infty} f(x, z) \delta(r-x \cos \varphi-z \sin \varphi) d x d z,
$$

где $\delta$ - дельта-функция, $r=x \cos \varphi+z \sin \varphi-$ нормальное уравнение прямой в плоскости $(x, z), r-$ перпендикуляр, опущенный из начала координат до этой прямой, $\varphi-$ угол между перпендикуляром и осью $x$, который принимает значения из интервала $[\pi / 2,+\pi / 2]$. Преобразование (1) известно как преобразование Радона [12].

Основой большинства алгоритмов томографии служит теорема о центральном сечении $[12,13]$, которую можно записать в виде

$$
P(\rho ; \varphi)=F(\rho \cos \varphi, \rho \sin \varphi),
$$

где заглавными буквами обозначены фурье-образы функций, заданных строчными буквами, т. е.

$$
\begin{gathered}
p(r ; \varphi)=\frac{1}{2 \pi} \int_{-\infty}^{\infty} P(\rho ; \varphi) e^{i \rho r} d \rho \\
f(x, z)=\frac{1}{4} \iint_{-\infty}^{\infty} F(u, w) e^{i(u x+w z)} d u d w
\end{gathered}
$$

$\rho, u, w-$ координаты, фурье-сопряженные соответственно пространственным координатам $r, x, z$, $i=\sqrt{-1}$. Теорему о центральном сечении (2) легко получить из выражения (1), выполнив преобразование Фурье над обеими его частями.

В локальной томографии в качестве проекционных данных используется не преобразование Радона (1), а его частная производная 1-го (иногда и 2-го) порядка по координате $r$ :

$$
g(r ; \varphi) \equiv \frac{\partial p(r ; \varphi)}{\partial r}=\frac{1}{2 \pi} \int_{-\infty}^{\infty} i \rho P(\rho ; \varphi) e^{i \rho r} d \rho .
$$

Выражение (5), полученное дифференцированием правой и левой частей выражения (3), назовем DICпроекцией. Сформируем из DIC-проекций (5) так называемое суммарное изображение:

$$
b(x, z)=\int_{-\pi / 2}^{+\pi / 2} g(x \cos \varphi+z \sin \varphi ; \varphi) d \varphi .
$$

Найдем связь суммарного изображения с исходной функцией $f(x, z)$. Подставляя в (6) выражение (5), получим

$$
b(x, z)=\frac{1}{2 \pi} \int_{-\pi / 2}^{\pi / 2} \int_{-\infty}^{\infty} i \rho P(\rho ; \varphi) e^{i \rho(x \cos \varphi+z \sin \varphi)} d \rho d \varphi .
$$

В алгоритмах реконструкции томограмм большую роль играет так называемый $\rho$-фильтр, пропускание которого пропорционально $|\rho|$. Поэтому для дальнейших преобразований воспользуемся простым соотношением:

$$
i \rho=-[-i \operatorname{sgn}(\rho)]|\rho|,
$$

где $\operatorname{sgn}(x)$ - знаковая функция:

$$
\operatorname{sgn}(x)= \begin{cases}1, & x>0 \\ -1, & x<0\end{cases}
$$

а фильтр с пропусканием $[-i \operatorname{sgn}(\rho)]$ соответствует преобразованию Гильберта [14]. Подставляя выражение (8) в (7) и используя теорему о центральном сечении (2), получим

$$
\begin{aligned}
b(x, z)= & -\frac{1}{2 \pi} \int_{-\pi / 2}^{\pi / 2} \int_{-\infty}^{\infty}[i \operatorname{sgn}(\rho)] F(\rho \cos \varphi, \rho \sin \varphi) \\
& \times e^{i \rho(x \cos \varphi+z \sin \varphi)}|\rho| d \rho d \varphi .
\end{aligned}
$$

Перейдем в полученном выражении от полярных к декартовым координатам путем замены $u=p \cos \varphi$, $w=p \sin \varphi$. Воспользуемся свойством знаковой функции $\operatorname{sgn}(\rho)=\operatorname{sgn}(\rho \cos \varphi)=\operatorname{sgn}(u)$ (для углов $\varphi$ в диапазоне $[-\pi / 2,+\pi / 2] \cos \varphi \geq 0)$, которое отмечено в работе [15], и получим

$$
b(x, z)=-\frac{1}{2 \pi} \int_{-\infty}^{\infty} \int_{-\infty}^{\infty}[-i \operatorname{sgn}(u)] F(u, w) e^{i(u x+w z)} d u d w .
$$

Используя запись преобразования Гильберта через преобразование Фурье [14], перепишем (9) в виде

$$
b(x, z)=-2 \pi H_{x}[f(x, z)],
$$

где $H_{x}$ - оператор преобразования Гильберта вдоль оси $x$.

Если зондирование производится в диапазоне углов $[0, \pi]$, то $\sin \varphi \geq 0$, поэтому используем другое преобразование:

$$
\operatorname{sgn}(\rho)=\operatorname{sgn}(\rho \sin \varphi)=\operatorname{sgn}(w) .
$$

Для этого диапазона углов зондирования получим

$$
b(x, z)=-2 \pi H_{z}[f(x, z)] .
$$

Впервые локальный алгоритм синтеза суммарного изображения из производных 1-го порядка был предложен И.М. Гельфандом и М.И. Граевым в 1991 г. [16]. 
В их работе было впервые показано, что такое суммарное изображение является преобразованием Гильберта от функции, описывающей объект. Для ограниченного диапазона углов зондирования выражения (10), (11) справедливы лишь в некотором приближении.

В заключение раздела подчеркнем, что для реконструкции изображений (10), (11) использовалась лишь одна операция суммирования по углу обратных DICпроекций без $\rho$-фильтрации. Поэтому данный алгоритм является локальным, и DIC-проекции могут быть ограниченными по размеру. Это главное достоинство полученных изображений.

Несмотря на то, что суммарные изображения (10), (11) не являются истинными томограммами, они несут полезную информацию об исследуемом объекте. Преобразование Гильберта подчеркивает границы резких перепадов плотности или показателя преломления (см. рисунки в следующем разделе).

Для реконструкции самой томограммы необходимо к выражениям (10), (11) применить еще раз преобразование Гильберта. Используя известное [17] выражение о том, что $H_{x}\left[H_{x}(f)\right]=-f$, получим

$$
f(x, z)=\frac{1}{2 \pi} H_{x}[b(x, z)] .
$$

Аналогичное выражение можно получить и для (11). Необходимо также отметить, что существует возможность восстановления локальных томограмм по суммарным изображениям тех же локальных областей. Так, из суммарного изображения (6) можно восстановить исходную функцию, применяя к (10) обратное преобразование Гильберта в ограниченных пределах интегрирования [18]. В настоящее время методы локальной томографии активно развиваются в медицинской томографии и фазоконтрастной томографии, основанной на синхротронном рентгеновском излучении[19].

\section{Нормализация DIC-проекций}

После получения DIC-проекций необходимо провести их нормализацию или согласование, что включает в себя две операции: нормировку проекций по „энергии“ и согласования проекций по центру тяжести.

Для обычных проекционных данных к первой процедуре согласования относится „энергетическая“ нормировка проекций, которая заключается в том, что нулевые моменты („энергии“) всех проекций должны быть равны между собой. Так как проекционные данные поступают с разных каналов, поэтому возможны ошибки, связанные с разбросом чувствительности каналов и погрешностями их калибровки. Устранение такого рода ошибок можно производить путем мультипликативной нормировки проекций, при которой их нулевые моменты приводятся либо к среднему, либо к заданному значению.

Вторая задача нормализации проекций - согласование систем координат, в которых определены проекции, и системы координат, относительно которой реконструируется объект [20]. Такое согласование осуществляется при помощи соотношения, связывающего первый момент объекта с первыми моментами его проекций. По этому соотношению центр тяжести проекций совпадает с проекцией на плоскость детектирования центра тяжести самого объекта. Таким образом, согласованные системы координат получаются при помещении начала координат для каждой проекции в ее центр тяжести.

Применим такой же подход к нормализации DICпроекций. Введем определения геометрических моментов $n$-го порядка от проекции (3) и DIC-проекции (5) следующим образом:

$$
\begin{aligned}
m_{n}(\varphi) & =\int p(r ; \varphi) r^{n} d r \\
m d_{n}(\varphi) & =\int g(r ; \varphi) r^{n} d r .
\end{aligned}
$$

Известно, что моменты функции $n$-го порядка определяются через производные $n$-го порядка от фурье-спектра функции на нулевой пространственной частоте $(\rho=0)$, T. e.

$$
m_{n}(\varphi)=(-i)^{-n} \frac{\partial^{n} P(\rho=0 ; \varphi)}{\partial^{n} \rho} .
$$

Отсюда следует

$$
\begin{gathered}
m_{0}(\varphi)=P(\rho=0 ; \varphi), \\
m_{1}(\varphi)=i \frac{\partial P(\rho=0 ; \varphi)}{\partial \rho}, \\
m_{2}(\varphi)=-\frac{\partial^{2} P(\rho=0 ; \varphi)}{\partial^{2} \rho} .
\end{gathered}
$$

Аналогично для фурье-спектра DIC-проекции,

$$
g(r ; \varphi)=\frac{1}{2 \pi} \int_{-\infty}^{\infty} G(\rho ; \varphi) e^{i \rho r} d \rho,
$$

получим выражения для геометрических моментов от DIC-проекций:

$$
\begin{gathered}
m d_{0}(\varphi)=G(\rho=0 ; \varphi), \\
m d_{1}(\varphi)=i \frac{\partial G(\rho=0 ; \varphi)}{\partial \rho}, \\
m d_{2}(\varphi)=-\frac{\partial^{2} G(\rho=0 ; \varphi)}{\partial^{2} \rho} .
\end{gathered}
$$

Для вычисления этих моментов воспользуемся тем, что фурье-спектр DIC-проекции $G(\rho ; \varphi)$ связан с фурьеспектром самой проекции $P(\rho ; \varphi)$ через выражение

$$
G(\rho ; \varphi)=i \rho P(\rho ; \varphi) .
$$

Используя это соотношение, получим

$$
m d_{0}(\varphi)=0,
$$




$$
\begin{aligned}
m d_{1}(\varphi)= & i \frac{\partial}{\partial \rho}(i \rho P(\rho=0 ; \varphi)) \\
= & i\left(i P(\rho=0 ; \varphi)+(\rho=0) i \frac{\partial P(\rho=0 ; \varphi)}{\partial \rho}\right) \\
= & -P(\rho=0 ; \varphi)=-m_{0}(\varphi), \\
m d_{2}(\varphi)= & -\frac{\partial^{2}}{\partial^{2} \rho}(i \rho P(\rho=0 ; \varphi)) \\
= & -i \frac{\partial}{\partial \rho}\left[P(\rho=0 ; \varphi)+\rho \frac{\partial P(\rho=0 ; \varphi)}{\partial \rho}\right] \\
& =-i\left[\frac{\partial P(\rho=0 ; \varphi)}{\partial \rho}+\frac{\partial P(\rho=0 ; \varphi)}{\partial \rho}\right. \\
& \left.+(\rho=0) \frac{\partial^{2}(P(\rho=0 ; \varphi))}{\partial^{2} \rho}\right] \\
= & -2 i \frac{\partial P(\rho=0 ; \varphi)}{\partial \rho}=-2 m_{1}(\varphi) .
\end{aligned}
$$

Таким образом, получили следующую связь между геометрическими моментами DIC -и простых проекций:

$$
\begin{gathered}
m_{0}(\varphi)=-m d_{1}(\varphi), \\
m_{1}(\varphi)=-\frac{1}{2} m d_{2}(\varphi) .
\end{gathered}
$$

Из (13) следует, что „энергия“ (момент нулевого порядка) всех DIC-проекций должна быть равна нулю.

Координата центра тяжести проекций определяется через геометрические моменты следующим соотношением:

$$
r_{(\text {ц.т. })}(\varphi)=\frac{m_{1}(\varphi)}{m_{0}(\varphi)} .
$$

Условие согласования проекций по центру тяжести гласит [20], что координата центров тяжести всех проекций должна быть одинакова. Это же условие должно выполняться и для DIC-проекций. Подставляя в (16) соотношения (14) и (15), получим

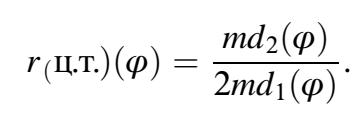

Логично объявить отношение моментов $\frac{m d_{2}(\varphi)}{2 m d_{1}(\varphi)}$ координатой центра тяжести DIC-проекции для угла зондирования $\varphi$, которая должна быть одинакова для всех проекций.

Помимо упомянутого согласования, на этапе предобработки проекционных данных производилось устранение фона. Из DIC-проекций вычиталась некоторая константа (вообще говоря, для каждой проекции своя), которая оценивалась приближенно по значениям вне изображения объекта.

\section{Компьютерное моделирование}

В дальнейших экспериментах в качестве тест-объекта использовались калиброванные шарики из диоксида кремния диаметром $5 \mu \mathrm{m}$ с показателем преломления $n=1.42$ на длине волны $650 \mathrm{~nm}$, помещенные в иммерсионную жидкость с показателем преломления $n_{0}=1.52$. Поэтому при проведении компьютерного моделирования с целью проверки правильности полученных выше формул был сформирован 2D-фантом в виде круга диаметром $5 \mu \mathrm{m}$, внутри которого показатель преломления был равен 0.1 , а вне круга -0 . Этот фантом имитирует центральное сечение шарика, погруженного в иммерсионную жидкость. На рис. $1, a, b$ приведены преобразование Гильберта от этого фантома и его сечения, полученные численным методом с использованием функции „hilbert“ в пакете Python (версия 3.6.3), в безразмерных величинах. Используя стандартную функцию „radon“, были получены обычные проекции фантома с шагом $3^{\circ}$ в диапазоне углов от $-90^{\circ}$ до $+90^{\circ}$, из которых численным дифференцированием были сформированы DIC-проекции. Используя функцию „iradon“ в режиме „unfiltered backprojection data“ (параметр „None“) в пакете Python, было восстановлено суммарное изображение фантома из DIC-проекций (рис. $1, c, d$ ) в безразмерных величинах. Согласно выражению (10) это изображение должно описываться одномерным преобразованием Гильберта от фантома вдоль горизонтальной оси. Сравнивая рис. $1, a, b$ и рис. $1, c, d$, видим заметное их сходство. Различия вызваны веерными артефактами за пределами объекта из-за конечного числа проекций. Применяя к полученному суммарному изображению обратное преобразование Гильберта (функция „ihilbert" пакета Python) согласно (12) получим, как и следовало ожидать, томограмму, представленную вместе с сечением на рис. $1, e, f$.

В заключение подчеркнем, что для восстановления полученных изображений использовались лишь две локальные операции - обратного проецирования и суммирования.

\section{Описание экспериментой установки}

В настоящей работе для проведения экспериментальных исследований метода локальной оптической томографии использовался оптический фазовый микротомограф на базе микроскопа проходящего типа с интерферометром бокового сдвига, оптическая схема которого представлена на рис. 2.

В качестве источника низкокогерентного излучения использовался точечный светодиод (pLED на рис. 2) фирмы Kyosemi Co., Япония, модель KED080RAXH, центральная длина волны излучения $650 \mathrm{~nm}$, полуширина спектра излучения $10 \mathrm{~nm}$, мощность излучения $0.6 \mathrm{~mW}$ при токе $25 \mathrm{~mA}$. В отличие от обычного точечный светодиод имеет небольшую площадку излучения (около $80 \mu \mathrm{m}$ ). Использование такого источника совместно с коллимирующим объективом CL (фокусное 

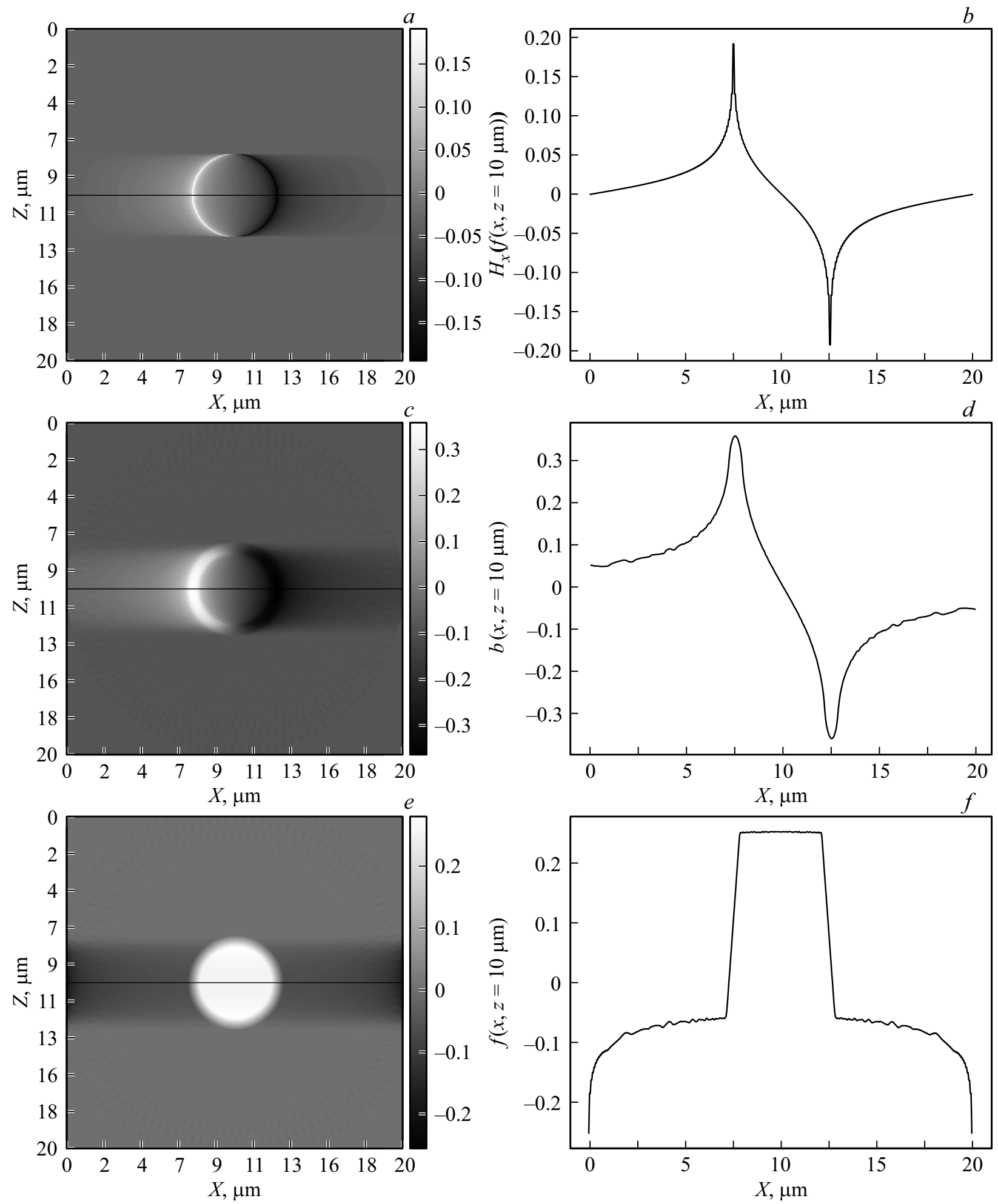

Рис. 1. Результаты компьютерного моделирования, серая шкала в безразмерных единицах: $a, b$ - гильберт-преобразование фантома и его сечение вдоль горизонтальной прямой; $c, d-$ суммарное изображение из DIC-проекций и его сечение вдоль горизонтальной прямой; $e, f$ - томограмма, полученная обратным гильберт-преобразованием от суммарного изображения, и ее сечение вдоль горизонтальной прямой.

расстояние $12 \mathrm{~mm}$ ) позволяет без потери мощности получить квазимонохроматическое излучение, имеющее высокую пространственную когерентность.
Для углового зондирования объекта применялся сканатор в виде одноосевого гальванического зеркала (GM на рис. 2) фирмы ThorLabs, модель GVS011. На ска- 


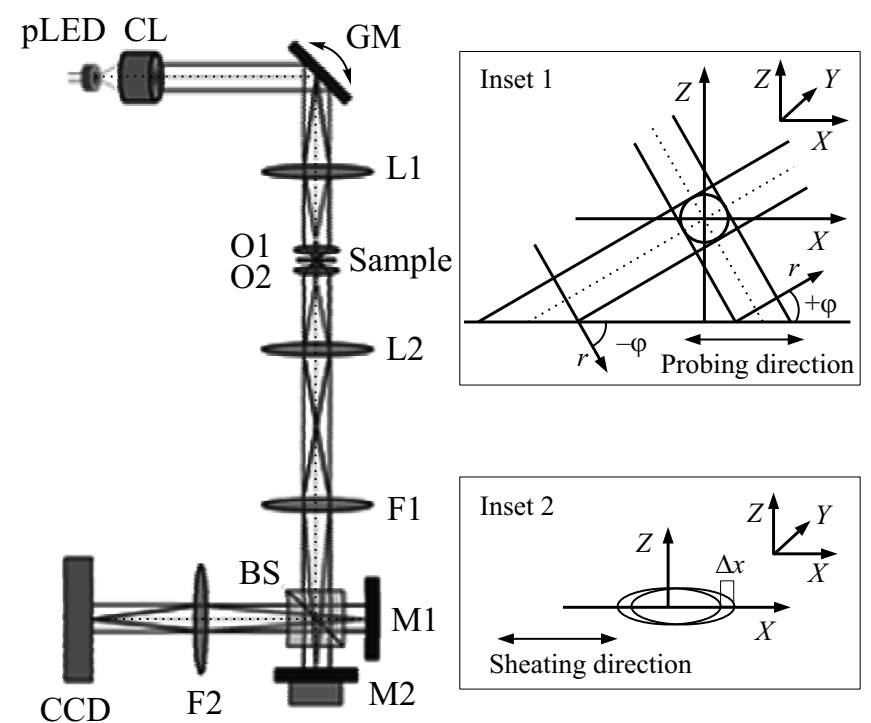

Рис. 2. Схема локального интерференционного томографического микроскопа: pLED - точечный светодиод; CL коллимирующий объектив; GM - гальваническое зеркало; L1, L2 - линзы; O1, O2 - микрообъективы; F1, F2 - фурьеобъективы; BS - светоделительный кубик; M1 - зеркало; M2 - зеркало на пьезоприводе; CCD - КМОП-камера; Inset 1, 2 - вставки 1, 2; Probing direction - направление зондирования; Shearing direction - направление сдвига.

натор подавалось напряжение в виде последовательности прямоугольных импульсов длительностью $100 \mathrm{~ms}$. Время, необходимое для того, чтобы зеркало перестало вращаться после изменения напряжения управляющего сигнала, составляет $0.4 \mathrm{~ms}$.

После отражения от зеркала GM плоский световой пучок фокусируется линзой L1 в передней фокальной плоскости микрообъектива O1 $(100 \times$, NA 1.30 , иммерсионный, UPlanFLN100X02, Olympus) и образует виртуальный точечный источник. Так как ось вращения гальванического зеркала GM находится в передней фокальной плоскости линзы L1, то при повороте зеркала GM этот точечный источник смещается в поперечной плоскости. В результате после микрообъектива O1 формируется узкий плоский пучок света, освещающий объект (Sample на рис. 2) под некоторым углом. Световое излучение, прошедшее через объект, собирается микрообъективом $\mathrm{O} 2$, который аналогичен микрообъективу О1. Угловой диапазон зондирования в томографической микроскопии зависит от числовой апертуры микрообъективов О1 и $\mathrm{O} 2$, и он составил $\pm 60^{\circ}$ для данных микрообъективов.

Исследуемый препарат состоит из двух покровных стекол толщиной $100 \mu \mathrm{m}$, разделенных тонкой прокладкой в $100 \mu \mathrm{m}$, между которыми находится объект в иммерсионной жидкости. Рабочий отрезок микрообъективов О1, О2 равен $200 \mu \mathrm{m}$, поэтому расстояние между микрообъективами составляет $400 \mu \mathrm{m}$ и его хватает для размещения препарата.

После микрообъектива О2 и фурье-объектива F1 световой пучок направляется в сдвиговый интерферометр
Майкельсона. Зеркала M1, M2 находятся в задней фокальной плоскости фурье-объектива F1, поэтому малый наклон зеркала M1 приводит только к поперечному сдвигу пучков после второго фурье.объектива F2 без их взаимного наклона. Следовательно, в такой схеме интерферометр настроен на бесконечно широкую полосу.

Восстановление фазовых изображений производится методом интерферометрии фазовых шагов по алгоритму Харихарана-Швайдера [21]. Для его реализации необходимо изменять оптическую длину пути одного из интерферирующих пучков, что в нашем приборе достигается непрерывным смещением зеркала М2 вдоль направления оптической оси. Это зеркало закреплено на многослойном пьезоактюаторе АПМ-2-7, состоящем из семи керамических блоков материала ЦТС-46 (,ЭЛПА“, Зеленоград), с помощью которого обеспечивается максимальное перемещение $14 \mu \mathrm{m}$ при напряжении $100 \mathrm{~V}$. Для непрерывного изменения оптической длины пути до величины, равной двум интерференционным полосам, на пьезоактюатор подается пилообразное напряжение амплитудой $5 \mathrm{~V}$ и периодом $200 \mathrm{~ms}$ (нарастание и спад). В течение нарастания или спада напряжения через равные промежутки времени производится регистрация девяти интерферограмм за время экспозиции $2 \mathrm{~ms}$.

Оси, вокруг которых выполняется поворот гальванического зеркала GM и M1, должны быть параллельными. Величина сдвига не превышает размер диска Эйри для данного типа микрообъективов. В этом случае сдвиговая интерферограмма будет нести информацию о производной 1-го порядка от проекции вдоль направления сканирования. Для регистрации сдвиговых интерферограмм использовалась монохромная КМОП-камера IDS UI3140CP-M-GL фирмы IDS GmbH, Германия. Эта камера позволяет регистрировать 10-bit изображения размером $1280 \times 1024$ pixels со скоростью $224 \mathrm{fps}$.

Синхронизация и управление гальваническим зеркалом, пьезоактюатором и КМОП-камерой осуществлялись от персонального компьютера с помощью платы L Card, модель L-502 (разработка OOO „Л Кард“, Москва). Эта плата представляет собой новую российскую систему сбора данных на основе интерфейса РСI Express современных материнских плат компьютеров. Собственное программное обеспечение WinPhast написано на языке $\mathrm{C}++$.

Экспериментально достигнуты следующие характеристики томографа: диапазон углов зондирования $\pm 60^{\circ}$, размер кадра $512 \times 512$ pixels, время съема данных для восстановления одной 2D-DIC-проекции $0.1 \mathrm{~s}$, время съема данных для восстановления 3D-томограммы по 60 2D-DIC-проекциям $6 \mathrm{~s}$. В качестве тест-объекта использовались калиброванные шарики из диоксида кремния диаметром $5 \mu \mathrm{m}$ (Product \#44054-5ML-F фирмы Sigma-Aldrich). На рис. 3, a показана центральная 2DDIC-проекция такого объекта под углом зондирования $\varphi=0^{\circ}$, при котором ось $r$ совпадает с осью $x$. Эта проекция представляет собой оптическую разность хода разность, нормированную на величину сдвига, и она 

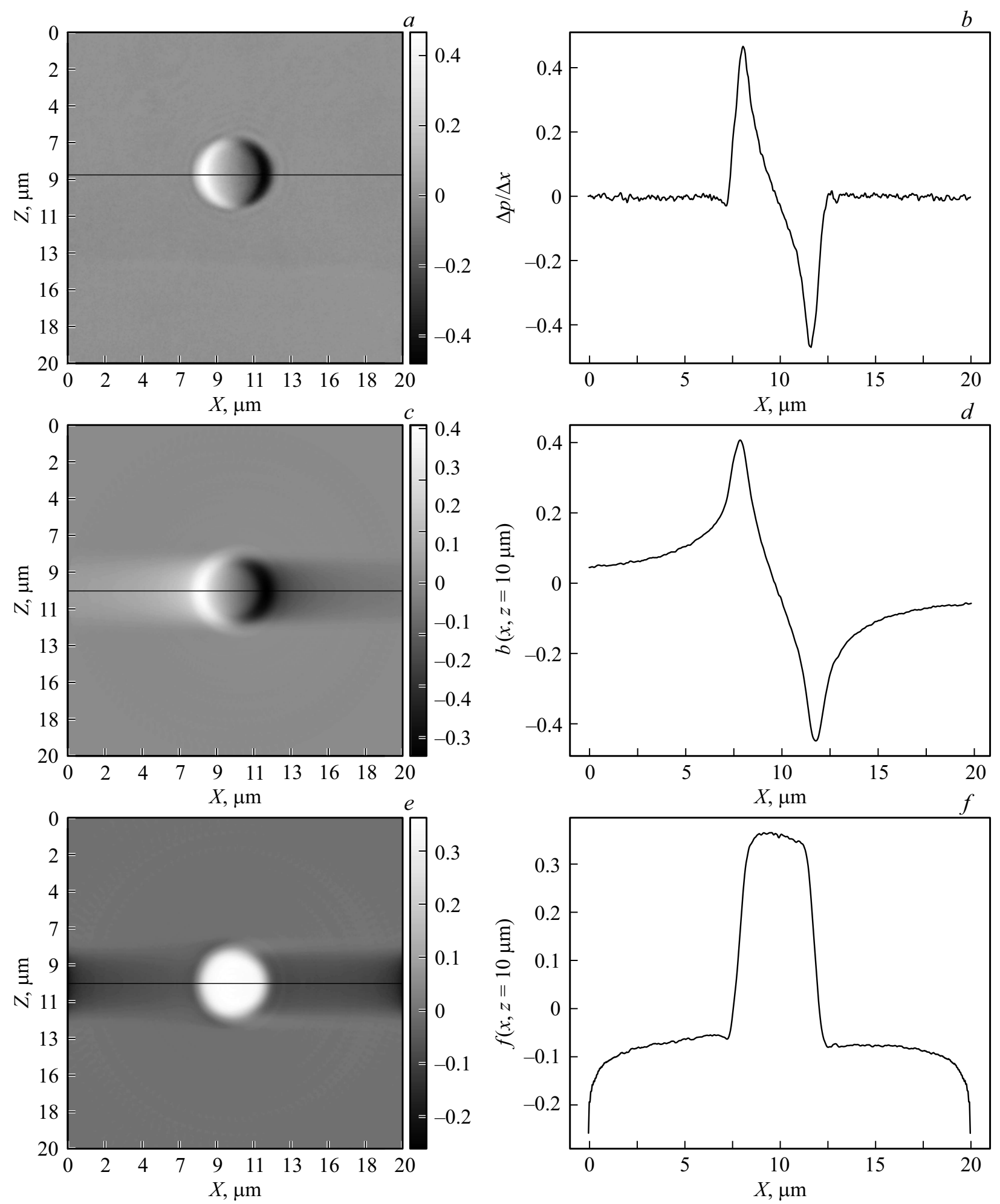

Рис. 3. Результат эксперимента с шариком из диоксида кремния диаметром $5 \mu \mathrm{m}$, серая шкала в безразмерных единицах: $a, b-2 \mathrm{D}-$ DIC-проекция и ее сечение вдоль горизонтальной прямой как 1D-DIC-проекция; $c, d-$ суммарное изображение из DIC-проекций и его сечение вдоль горизонтальной прямой; $e, f$ - томограмма, полученная обратным гильберт-преобразованием суммарного изображения, и ее сечение вдоль горизонтальной прямой.

описывается выражением

$g(x ; \varphi) \equiv \frac{\partial p(x ; \varphi}{\partial x} \approx \frac{\Delta p(x ; \varphi)}{\Delta x}=\frac{(p(x ; \varphi)-p(x+\Delta x ; \varphi)}{\Delta x}$, где $\Delta x=700 \mathrm{~nm}$ - величина сдвига, которая была реализована в экспериментах. Таким образом DIC-проекция является безразмерной величиной, которая приближен- 
но описывается частной производной 1-го порядка от оптической разности хода в горизонтальном направлении.

На полученной 2D-DIC-проекции было выбрано сечение, отмеченное на рис. 3, a горизонтальной прямой, вдоль которого была выделена 1D-DIC-проекция (рис. 3,b). Так как исследуемый объект является шариком, то все его боковые проекции должны быть одинаковы. Поэтому для реконструкции томограмм мы использовали одну эту 1D-DIC-проекцию, размноженную на весь диапазон углов $\pm 90^{\circ}$. Такая процедура была выбрана для того, чтобы сравнить результат реконструкции томограммы по экспериментальным проекциям с результатами компьютерного моделирования.

Суммарное изображение, восстановленное из шестидесяти таких 1D-DIC-проекций, приведено на рис. 3,c вместе с сечением на рис. $3, d$. Обратное преобразование Гильберта от полученного суммарного изображения приводит к восстановлению томограммы центрального сечения шарика, которая вместе с сечением приведены на рис. $3, e, f$. Сравнение результатов моделирования на рис. 2 с результатами эксперимента на рис. 3 показывает их хорошее сходство.

\section{Заключение}

Исследован метод локальной томографии по дифференциальным проекциям, представляющим собой частные производные от обычных проекций. Такие проекции легко получить в оптическом томографе методами DICконтраста и сдвиговой интерферометрии. Преимущество локальной томографии состоит в том, что она дает возможность быстрого вычисления искомого распределения в интересующей точке или области без проведения полной реконструкции всего сечения.

Показано, что суммарное изображение из DICпроекций пропорционально преобразованию Гильберта от исходной функции, описывающей объект, а применение к этому изображению обратного преобразования Гильберта ведет к восстановлению томограммы объекта.

Проведено компьютерное моделирование на фантоме, моделирующем сечение шарика, которое подтвердило, что суммарное изображение из DIC-проекций, согласно выражению (10), описывается преобразованием Гильберта от фантома. Обратное преобразование Гильберта от полученного изображения позволяет восстановить, согласно выражению (12), томограмму фантома.

Для экспериментальной проверки результатов моделирования был собран макет локального интерференционного томографического микроскопа, на котором были получены DIC-проекции прозрачного шарика из диоксида кремния. Впервые предложена и использована процедура нормализации проекционных данных по геометрическим моментам от DIC-проекций.

Для адекватного сравнения с результатами компьютерного моделирования проекционные данные были размножены на полный диапазон углов $\pm 90^{\circ}$, по которым были восстановлены суммарное изображение и томограмма центрального сечения шарика. Сравнение результатов моделирования с результатами эксперимента показало их хорошее сходство.

Описанный и исследованный в настоящей работе томографический микроскоп может быть использован для исследования внутренней структуры различных прозрачных объектов, в том числе изделий, изготовленных методами аддитивных технологий из кварцевого стекла или прозрачного полимера. В дальнейшем предполагается также использовать методы локальной томографии для исследования динамических процессов внутри живых клеток. Эти процессы могут быть вызваны пространственным смещением отдельных органелл клетки, а также изменением пространственного распределения показателя преломления внутри клетки, обусловленного синтезом белка в клетках, их делением, воздействием внеклеточных факторов различной природы. Локальная томография позволяет оценить динамику изменения сухой массы клеток, плотности сухой массы, цитоплазматического объема, сферичности и локальных вязкоупругих свойств цитоплазмы исследуемых живых объектов.

Работа была выполнена при финансовой поддержке Министерства образования и науки Российской Федерации в рамках выполнения соглашения № 14.625.21.0041 от 26.09.2017 г. (уникальный идентификатор прикладных научных исследований RFMEFI62517X0041).

Часть исследований выполнена с привлечением оборудования Центра коллективного пользования уникальным научным оборудованием в области нанотехнологий (ЦКП МФТИ).

\section{Список литературы}

[1] Klein J. et al. // 3D Printing and Additive Manufacturing. 2015. V. 2. N 3. P. $92-105$.

[2] Sava O.J. et al. // Opt. Engineering. 2018. V. 57. N 4. P. 041402.

[3] Luo J. et al. // Opt. Engineering. 2018. V. 57. N 4. P. 041408.

[4] Вишняков Г.Н., Левин Г.Г. // Опт. и спектр. 1998. Т. 85. № 1. C. 82.

[5] Lauer V. // Proc. SPIE. 2000. V. 4164. P. 122.

[6] Vishnyakov G.N., Levin G.G.,. Minaev V.L, Pickalov V.V., Likhachev A.V. // Microscopy and Analysis. 2004. V. 18. N 1. P. 19.

[7] Sung Y., Choi W., Fang-Yen C., Badizadegan K., Dasari R.R., Feld M.S. // Opt. Express. 2009. V. 17. N 1. P. 266.

[8] Shin S., Kim K., Yoon J., Park Y.-K. // Opt. Letters. 2015. V. 40. N 22. P. 5407.

[9] Kuś A., Krauze W., Kujawińska M. // J. Biomed. Optics. 2015. V. 20. N 11. P. 111216.

[10] Пуро А.Э., Каров Д.Д. // Опт. и спектр. 2018. Т. 124. № 5. C. $700-705$.

[11] Вишняков Г.Н., Левин Г.Г., Минаев В.Л., Некрасов Н.А. // Опт. и спектр. 2016. Т. 121. № 6. С. 1020.

[12] Хермен Г. Восстановление изображений по проекциям: основы реконструктивной томографии. М.: Мир, 1983. $352 \mathrm{c}$. 
[13] Левин Г.Г., Вишняков Г.Н. Оптическая томография. М.: Радио и связь, 1989. 224 с.

[14] Папулис A. Теория систем и преобразований в оптике. М.: Мир, 1971. 495 c.

[15] Noo F., Clackdoyle R., Park J.D. // Phys. Med. Biol. 2004. V. 49. P. 3903.

[16] Гельфанд И.М., Граев М.И. // Функциональный анализ и его приложения. 1991. Т. 25. № 1. С. 1.

[17] Titchmarsh E. Introduction to the theory of Fourier integrals (2nd ed.). Oxford University: Clarendon Press, 1986.

[18] Anastasio M.A., Pan X. // Opt. Letters. 2007. V. 32. N 21. P. 3167.

[19] Sunaguchi N., Yuasa T., Gupta R., Ando M. // Appl. Phys. Letters. 2015. V. 107. N 25. P. 253701.

[20] Пикалов В.В., Мельникова Т.С. Томография плазмы. Новосибирск: Наука, 1995. 229 с.

[21] Malacara D., Servin M., Malacara Z. Interferogram Analysis for Optical Testing (2nd ed.).CRC Press, 2005. 